\title{
Validação Brasileira da Escala de Autopercepção de Harter para Crianças
}

\author{
Brazilian Validity of the Harter'Self-perception Profile for Children
}

\author{
Nadia Cristina Valentini ${ }^{*}, a$, Gabriela Villwock ${ }^{b}$, Lenamar Fiorese Vieira $^{c}$, \\ José Luiz Lopes Vieira ${ }^{c}, \&$ Marcus Levi Lopes Barbosa ${ }^{a}$ \\ ${ }^{a}$ Universidade Federal do Rio Grande do Sul, Porto Alegre, Brasil, \\ ${ }^{b}$ Escola Estadual de Ensino Médio Amado Rossi, Porto Alegre, Brasil \\ \& 'Universidade Estadual de Maringá, Maringá, Brasil
}

\begin{abstract}
Resumo
O Self-perception Profile for Children (SPPC) é usado para investigar a autopercepção de crianças. O objetivo deste estudo foi traduzir o SPPC para o português e verificar a validade dos itens, da análise fatorial confirmatória e da consistência interna teste-reteste dessa versão. Participaram do estudo, além de sete profissionais, 746 crianças ( 8 a 12 anos), as quais responderam o instrumento em dois momentos. Os resultados indicam que a versão brasileira contém questões claras e pertinentes $(\mathrm{CVCt}>0,70)$ e apresenta índices satisfatórios de validade fatorial confirmatória $(\chi 2 / \mathrm{gl}=4,33$; Non-normed Fit Index $=$ 0,958; Comparative Fit Index =0,967 e Tucker e Lewis's Index of Fit $=0,962$ ) e de consistência interna teste-reteste, e assim a versão brasileira se mostrou válida.

Palavras-chave: Escala de Autopercepção; Validação; Autoconceito; Crianças.
\end{abstract}

\begin{abstract}
Harter's Self-perception Profile for Children (SPPC) is used to evaluate children's self-worth. The objective of this study was to translate and verify the validity of the items, the confirmatory factorial analysis and the test-retest internal consistence of the Brazilian version of SPPC. The participants of this study were 7 professionals and 746 children ( 8 to 12 years old) who answered the instrument at two different moments. The results indicated that the Brazilian version contains clear and pertinent questions $(\mathrm{CVCt}>0.70)$, shows satisfactory indices of the confirmatory factorial validity $(\chi 2 / \mathrm{gl}=4.33$; Nonnormed Fit Index $=0.958$; Comparative Fit Index $=0.967$ and Tucker and Lewis's Index of Fit $=0.962$ ) as well as of internal consistence. Thus, the Brazilian version showed to be valid.

Keywords: Self-perception Scale; Validity; Self-worth; Children.
\end{abstract}

Ao longo de seu desenvolvimento a criança é compelida a relacionar-se efetivamente com o meio em que vive, e o faz pelo desejo de, com sucesso, exercer influência sobre ele. A competência percebida, julgamento expressado pela criança sobre suas habilidades em experiências de conquista, é considerada um importante mediador da motivação infantil, incidindo de maneira decisiva na formação do autoconceito (Harter, 1985, 1999). Ao desenvolver-se cognitivamente, a criança passa a fazer distinção entre o conceito de habilidade (capacidade de executar a tarefa com maestria) e o de esforço (energia necessária para desenvolver mestria), estabelecendo relações entre suas percepções e a dificuldade da tarefa e alterando as suas percepções de competência de acordo com as experiências vividas (Harter, 1999; Valentini \& Rudisill, 2004).

* Endereço para correspondência: Universidade Federal do Rio Grande do Sul, Escola Superior de Educação Física, Rua Felizardo 750, LAPEX sala 106b, Jardim Botânico, Porto Alegre, RS, Brasil, CEP 90690-200. E-mail: nadiacv@esef.ufrgs.br
Diferentes critérios são empregados pela criança no julgamento de suas competências. De início predominam critérios da cultura familiar e posteriormente a cultura dos pares elege parâmetros e valores que são incorporados pela criança. Não obstante, a expressão dos próprios julgamentos não necessariamente é precisa. Ao avaliar sua competência, a criança pode se perceber competente em um domínio (cognitivo, social e/ou físico) e evidenciar um desempenho real não condizente com a sua percepção; ou ainda, perceber-se pouco competente para determinada tarefa e ao realizá-la obter sucesso efetivo, embora não seja capaz de entender as razões deste sucesso (Harter 1999; Valentini \& Rudisill, 2004; Wu, 2003).

Ao superestimar a competência, a criança cria expectativas não realistas de desempenho futuro. Vivenciar fracassos quando a tarefa é percebida como fácil resulta em baixa percepção de competência e no abandono do desafio. Ao subestimar sua atual competência, a criança elabora baixas expectativas em relação à sua competência futura, o que repercute negativamente no seu desempenho e na motivação para persistir nas atividades, tor- 
nando-a mais fragilizada no processo de aprendizagem (Chen et al., 2004; Harter, 1985, 1999; Valentini \& Rudisill, 2004).

Estudos sugerem que baixas percepções de competência estão associadas com motivação intrínseca fragilizada (Hassandra, Goudas, \& Chroni, 2003; Wu, 2003), desempenho escolar inadequado (Altermatt \& Pomerantz, 2003; Chen et al., 2004; Valentini \& Rudisill, 2004), dificuldades motoras (Valentini \& Rudisill, 2004), déficit de atenção e dificuldades de aprendizagem (Barber, Grubbs, \& Cottrell, 2005; Heath \& Glen, 2005), obesidade (Franklin, Denyer, Steinbeck, Caterson, \& Hill, 2006), baixa estatura (Van Pareren, Duivenvoorden, Slijper, Koot, \& Hokken-Koelega, 2004), doenças crônicas (Chernoff, Ireys, DeVet, \& Kim, 2002), agressão, comportamentos antissociais e delinquência (Donnellan, Trzesniewski, Robins, Moffitt, \& Caspi, 2005).

Nos mais variados contextos, a competência percebida é mediadora de novas conquistas e essencial para o desenvolvimento integral da criança. A avaliação de como a criança se percebe é de fundamental importância para a ação de profissionais e familiares. O conhecimento da autopercepção da criança permite que sejam implementados experiências, instruções e reforços apropriados aos seus níveis de desenvolvimento, auxiliando-a na construção de percepções de competência reais e de um autoconceito positivo. Para entender este importante processo de desenvolvimento e estabelecer parâmetros efetivos de conquistas individuais se faz necessário o uso de instrumentos de diagnóstico adequados e validados.

Esta preocupação tem sido observada na literatura. Uma iniciativa recente foi a validação do instrumento de autopercepção de adolescentes (Harter, 1985) realizada por Bandeira, Arteche e Reppold (2008). Essas autoras avaliaram a validade concorrente e a consistência interna e destacaram a importância do uso desta escala para adolescentes brasileiros, enfatizando a boa qualidade psicométrica do instrumento e a adequação dos índices de consistência interna ( $\alpha=0,60$ a $\alpha=0,88)$ obtidos em uma amostra no Sul do Brasil. Com relação a autopercepção de crianças, a maioria dos estudos atuais conduzidos nas diversas áreas (enfermagem, fisioterapia, psicologia, educação) utiliza como instrumento de diagnóstico o Self Perception Profile for Children (Harter, 1985). No Brasil, este instrumento tem sido utilizado por vários pesquisadores (Chen et al., 2004; Fiorese, 1993; Valentini \& Rudisill, 2004; Vieira, Krebs, \& Vieira, 1997). Esses pesquisadores reconhecem a validade lógica do instrumento e o traduziram para o português; mas o utilizaram sem a validação, necessária para conferir aos dados obtidos a confiabilidade imprescindível à pesquisa. Embora esses estudos tenham repercutido de forma relevante na prática e na pesquisa, restam importantes questões relacionadas à adequação do uso do instrumento para crianças brasileiras. $\mathrm{O}$ uso de instrumento não validado compromete sua eficácia diagnóstica para outras culturas que não a de sua origem (Cronbach, 1996; Harter, 1999; Vallerand, 1989).

Em se tratando de pesquisa, embora a validade lógica seja importante, pesquisadores necessitam de evidências mais objetivas em um instrumento avaliativo. Observase que ao longo dos anos diferentes abordagens de validação têm sido adotadas por pesquisadores. Nos anos 70 (American Educational Research Association [AERA], American Psychological Association [APA], \& National Council on Measurement in Education [NCME], 1974) fortaleceu-se a tendência de utilizar como evidências da validade a serem observadas em um estudo aquelas relativas ao critério, conteúdo e construto; perspectiva amplamente difundida no Brasil (Pasquali, 1999, 2003). Esta concepção tem sido quase exaustivamente discutida, sendo considerada fragmentada e incompleta (Urbina, 2004), de forma que a noção de validade tem avançado, incluindo, além das evidências relativas ao critério, conteúdo e construto, todas as possíveis evidências que corroborem a interpretação pretendida dos escores de um teste, tendo-se em vista a sua finalidade. Essas novas metodologias de validação exigem uma ampla fonte de evidências para que uma medida seja considerada válida (AERA, APA, \& NCME, 1999; Berry, Poortinga, Segall, \& Dasen, 1992; Urbina, 2004; Vallerand, 1989). Ao se ampliar o número de evidências indicadoras da validade, os pesquisadores podem utilizar com mais segurança diferentes instrumentos em outras culturas que não a sua de origem. Em relação ao Self Perception Profile for Children, a própria autora do instrumento fortemente sugere que, em uma perspectiva cross-cultural, pesquisadores necessitam determinar se o conteúdo deste instrumento, criado na cultura norte-americana, é relevante na cultura em que será utilizado (Harter, 1999).

Este estudo teve como objetivos: (a) fazer uma dupla tradução do Self Percepcion Profile for Children (SPPC) para a língua portuguesa: a tradução reversa e a independente; (b) verificar, segundo o parecer de juízes especificamente designados para a tarefa, a validade dos itens do SPPC quanto à clareza e à pertinência; (c) avaliar a fidedignidade do SPPC para uma população do Sul do Brasil por meio de teste-reteste; (d) avaliar a validade fatorial confirmatória do SPPC para uma população do Sul do Brasil.

\section{Método}

\section{Participantes}

Participaram deste estudo sete profissionais (quatro tradutores juramentados e três doutores em desenvolvimento humano), os quais aceitaram, de forma livre e esclarecida, participar dos processos de tradução, adaptação e validação de conteúdo do SPPC (Harter, 1985). $\mathrm{O}$ número de tradutores e juizes participantes segue as recomendações de Hernandez-Nieto (2002) e Vallerand (1989) para processos de validação transcultural de 
Valentini, N. C., Villwock, G., Vieira, L. F., Vieira, J. L. L. \& Barbosa, M. L. L. (2010). Validação Brasileira da Escala de Autopercepção de Harter para Crianças.

instrumentos. Participaram também 746 crianças, provenientes de 21 escolas do Estado do Rio Grande do Sul e 12 escolas Paraná (ensino infantil e fundamental). Um grupo de 25 crianças de ambos os sexos, aleatoriamente selecionadas e estratificadas por gênero e idade (5 crianças em cada grupo de idade), avaliou as questões do instrumento no que diz respeito à linguagem e à forma como o conteúdo estava sendo apresentado (validade aparente ou de face); outro grupo, com 721 crianças, respondeu ao instrumento final. O tamanho da amostra foi determinado com base nas recomendações de Dassa (1999), que propõe, no mínimo, 10 participantes por item do instrumento. Como se pretendia verificar a validade em cada um dos sexos, foi necessário um mínimo de 360 sujeitos em cada sexo. Das crianças (entre 8 e 12 anos), 360 eram do sexo masculino e 361 do sexo feminino, sendo distribuídas da seguinte forma: 8 anos $(n=170 ; 23,6 \%), 9$ anos $(n=184 ; 25,5 \%), 10$ anos $(n=197 ; 27,3 \%), 11$ anos $(n=97 ; 13,5 \%), 12$ anos $(n=73 ; 10,1 \%)$. Quanto à etnia, $433(60 \%)$ foram identificadas por seus pais e/ ou responsáveis legais, por meio de um questionário sociodemográfico, como brancas; 206 (29\%) como pardas; 61 (8\%) como negras; e 21 (3\%) como orientais. Do estudo participaram somente crianças cujos pais ou re- presentantes legais assinaram o Termo de Consentimento Livre e Esclarecido e que manifestaram verbalmente seu desejo de participar. Esta pesquisa foi aprovada pelo Comitê de Ética da Universidade Federal do Rio Grande do Sul ([UFRGS], processo n. ${ }^{\text {o }}$ 2008018).

\section{Instrumentos}

No presente estudo foi utilizado o SPPC (Harter, 1985), instrumento que avalia a competência percebida de crianças de 8 a 12 anos. O instrumento contém seis subescalas (que podem ser usadas separadamente) em cinco domínios específicos de competência: competência escolar, aceitação social, competência atlética, aparência física e conduta comportamental, além da subescala do autoconceito global, que reflete as diferenças dos indivíduos nos vários domínios, proporcionando uma imagem detalhada do autoconceito. Cada subescala do Self Perception Profile for Children contém seis questões, constituindo o total de 36 questões, organizadas em estrutura de respostas alternativas na escala do tipo Likert de 1 a 4 pontos, na qual a criança identifica quanto cada item é percebido como realmente verdadeiro ou parcialmente verdadeiro para ela. Complementarmente, utilizou-se um questionário sociodemográfico simples contendo informações sobre idade, sexo e etnia.

Tabela 1

Escalas do Instrumento Self Perception Profile for Children

\begin{tabular}{lrl}
\hline Escalas & Questões do SPPC & Competência \\
\hline Competência Escolar & $1,7,13,19,25,31$ & · Percepção das competências cognitivas relacionadas à escola. \\
Aceitação Social & $2,8,14,20,26,32$ & $\cdot$ Percepção do grau de relacionamento das crianças com amigos. \\
Competência Atlética & $3,9,15,21,27,33$ & · Percepção quanto à realização de atividades esportivas e ao ar livre. \\
Aparência Física & $4,10,16,22,28,34$ & · Percepção do grau de satisfação das crianças com tipo de corpo. \\
Conduta comportamental & $5,11,17,23,29,35$ & · Percepção de o comportamento ser adequado ou problemático \\
Autoconceito & $6,12,18,24,30,36$ & · Percepção das crianças do quanto gostam delas própria como pessoa.
\end{tabular}

Utilizou-se também a Escala de Clareza e Pertinência do tipo Likert (em 5 pontos), a qual foi respondida pelos juízes avaliadores a fim de avaliar a pertinência teórica e a clareza na linguagem do instrumento. Estas escalas permitiram investigar a consistência no julgamento das opiniões dos juízes avaliadores quanto aos aspectos relativos às questões do instrumento, partindo de "pouquíssima pertinência/clareza" (resposta 1) até "muitíssima pertinência/clareza" (resposta 5).

\section{Procedimentos}

Requereu-se à autora do teste, professora $\mathrm{PhD}$. Susan Harter, autorização formal para tradução e uso deste instrumento no Brasil. Tradutores juramentados foram contratados para traduzir o instrumento, e para avaliar a clareza e pertinência dos itens do instrumento foram contratados doutores em desenvolvimento humano. O trabalho desse grupo de especialistas foi inicialmente individualizado e posteriormente realizado em conjunto.
Quanto à tradução dupla (reversa e independente), primeiramente, dois tradutores de língua materna portuguesa, contratados de forma independente, sem saberem que outro profissional estava engajado no mesmo trabalho, traduziram para o português o SPPC; em um segundo momento dois outros tradutores foram contratados, desta vez de língua materna inglesa, para reverterem para o inglês as versões traduzidas para o português.

Quanto à participação de juízes avaliadores independentes, doutores (professores universitários) em desenvolvimento humano, atuantes em Psicologia, foram convidados a participar para, no papel de juiz avaliador, verificar a adequação das questões da versão original do SPPC. A função dos profissionais foi responder e pontuar na escala Likert se, de acordo com sua experiência e conhecimento teórico, crianças de 8 a 12 anos compreenderiam a estrutura de linguagem da questão (clareza) e se a questão media o construto teórico que ela se propunha a avaliar (pertinência). Os profissionais foram 
também solicitados a sugerir modificações de termos ou de estruturas fraseológicas, com vista a avaliar se o item traduzido e adaptado fora formulado de modo que não parecesse ridículo ou despropositado, no entendimento das crianças. As discordâncias ficaram restritas a termos muito semelhantes, as quais foram discutidas em comitê (um dos autores e três juízes). Em consenso foi incorporado o termo que melhor descrevesse o objetivo do item, considerando-se a faixa etária em foco.

Quanto aos procedimentos de avaliação da clareza pelo público-alvo, a versão em português foi aplicada para testar a versão brasileira experimental e avaliar se os termos eram claros e sem ambigüidades e se a linguagem era acessível e adequada, do modo que não parecesse risível ou despropositada para as crianças. Crianças $(n=25)$ com idades entre 8 e 12 anos (sendo 5 para cada faixa etária, em conformidade com o que recomenda a autora do instrumento) responderam todas as questões. As crianças foram reunidas em uma sala de aula, onde lhes foram apresentadas as instruções sobre o instrumento. Após a leitura de cada questão, feita em voz alta pelo pesquisador, as crianças foram questionadas quanto à compreensão da questão no tocante ao vocabulário e ao sentido de cada uma delas. Algumas crianças perguntaram o significado de algumas palavras e o sentido de algumas frases. Sempre que questionamentos eram realizados, o pesquisador (a) explicava o significado da palavra e/ou questão; e (b) solicitava que as crianças expressassem com suas próprias palavras o que tinham compreendido. Este procedimento foi repetido posteriormente.

Para a aplicação do instrumento às crianças participantes $(n=746)$ foram contatados diretores e coordenadores de escolas do Rio Grande do Sul e do Paraná. Nesse contato o pesquisador explicou o objetivo da pesquisa e os procedimentos metodológicos que seriam adotados. Às escolas que aceitaram participar do estudo foram enviadas, para serem encaminhadas aos pais ou responsáveis, cópias do termo de consentimento, em que se lhes assegurava o anonimato dos dados. A aplicação do instrumento foi realizada nas escolas de origem das crianças, em pequenos grupos. As crianças foram instruídas quanto à forma de responder às questões do instrumento, e exemplos de questões foram lidos em voz alta; porém cada criança respondeu individualmente ao instrumento, levando para isso entre 30 e 40 minutos. Entre 7 e 10 dias após a testagem inicial, foi realizada a retestagem, nos mesmos moldes da primeira avaliação.

\section{Análise dos Dados}

Os dados foram avaliados com o auxílio dos softwares SPSS (versão 13.0 para Windows) e Amos (versão 4.0 para Windows). Seguindo-se os princípios norteadores comumente aceitos na literatura especializada (Bryman \& Cramer, 1999; Cronbach, 1996; Vallerand, 1989), foram utilizados o Cálculo de Coeficiente de Validade de Conteúdo com ponto de corte de 0,7 , uma vez que envolvia juízes com formações e experiências diferenciadas
(Kline \& Saggino, 1995), correlações e teste $t$ pareado nos dados de teste-reteste e a análise fatorial confirmatória.

\section{Resultados}

\section{Dupla Tradução Reversa e Independente e Adaptação do Instrumento}

A tradução do SPPC para a língua portuguesa foi realizada por meio da tradução invertida, que envolveu quatro tradutores. Dois tradutores bilíngues realizaram a tradução do instrumento da língua inglesa para a portuguesa, do que resultaram duas traduções independentes, bastante próximas, mas não exatamente iguais. As versões em português foram reconvertidas para a língua inglesa por outros dois tradutores, sem a ajuda da versão original. Assim foram obtidas quatro versões, duas em cada língua.

Após a tradução inversa, os quatros tradutores se reuniram em comissão para realizar a avaliação e as modificações das versões preliminares. As duas traduções para a língua inglesa foram comparadas com a versão original do instrumento, a semântica das questões foi mantida e as poucas mudanças necessárias restringiram-se à substituição de palavras pouco usadas por sinônimos mais frequentes no cotidiano. As duas versões na língua portuguesa foram revisadas. Correções em relação aos termos técnicos utilizados na tradução e adequação dos itens para a compressão pelo público-alvo (crianças de 8 a 12 anos) constituíram o foco das discussões da comissão de tradutores. As versões em língua portuguesa foram unificadas, resultando na versão final do instrumento, a Escala de Autopercepção para Crianças (EAPC).

\section{Avaliação do Instrumento pelo Público-Alvo}

A EAPC foi aplicada experimentalmente a 25 crianças da população-alvo do instrumento. A partir de suas respostas, 7 das 36 questões foram reescritas com o objetivo de melhorar sua compreensibilidade, sendo repetido o mesmo procedimento após as questões serem reescritas sem que ficassem dúvidas para as crianças.

\section{Validação de Conteúdo por Juízes}

Com os dados obtidos nas respostas dos juízes avaliadores foram calculados os coeficientes de validade de conteúdo (CVC) para os dois aspectos em análise: clareza e pertinência (ver Tabela 2) da EAPC (Kline \& Saggino, 1995). Na subescala "competência escolar" obteve-se resultado limítrofe $(\mathrm{CVCt}=0,68)$.

\section{Avaliação da Fidedignidade Teste-Reteste}

Estatísticas preliminares foram conduzidas para observar a confiabilidade dos valores das médias. As médias das questões variaram de 2,29 a 3,35 (amplitude de 1 a 4). O mesmo ocorreu com as dimensões da escala, em que as médias variaram de 16,45 a 18,51 (amplitude de 6 a 36). As estatísticas preliminares permitiram adotar 
Valentini, N. C., Villwock, G., Vieira, L. F., Vieira, J. L. L. \& Barbosa, M. L. L. (2010). Validação Brasileira da Escala de Autopercepção de Harter para Crianças.

Tabela 2

Coeficientes de Validade de Conteúdo para os Critérios Clareza e Pertinência

\begin{tabular}{lcc}
\hline \multicolumn{1}{c}{ Escalas } & \multicolumn{2}{c}{$\mathrm{CVC}_{\mathrm{t}}$} \\
& Clareza & Pertinência \\
\hline Competência escolar & 0,68 & 0,86 \\
Aceitação social & 0,91 & 0,89 \\
Competência atlética & 0,77 & 0,86 \\
Aparência física & 0,86 & 0,89 \\
Conduta comportamental & 0,79 & 0,88 \\
Autoconceito & 0,85 & 0,89 \\
Escala Total (SPPC) & 0,79 & 0,88 \\
\hline
\end{tabular}

dois procedimentos utilizados na avaliação da fidedignidade por meio de teste e reteste: correlações e teste $t$ pareado.

O resultado da correlação de Pearson entre os escores do teste-reteste na escala total foi positiva, forte $(r=0,83)$ e altamente significativa $(p<0,01)$. Nas subescalas, obtiveram-se valores altamente significativos $(p<0,01)$, com índices de correlação variando de 0,54 a 0,70 . Os resultados do teste $t$ pareado evidenciam que somente uma subescala, competência escolar, apresentou resultados significativos entre o teste e reteste. Os resultados das correlações questão-questão competência escolar ( $\mathrm{r}_{\text {mediana }}$ $=0,18)$, aceitação social $\left(r_{\text {mediana }}=0,11\right)$, competência atlética $\left(r_{\text {mediana }}=0,14\right)$, aparência física $\left(r_{\text {mediana }}=0,27\right)$, conduta comportamental $\left(r_{\text {mediana }}=0,18\right)$ e autoconceito $\left(r_{\text {mediana }}=0,20\right)$ indicam correlações significativas, porém baixas, em quase todas as dimensões. (Tabela 3 ).

As correlações teste-reteste por grupo de idade foram positivas e altamente significativas, apresentando valores mais baixos nos grupos de menor idade. Os valores do teste $t$ pareado nas diferentes idades apresentaram diferenças significativas restritas à subescala da competência escolar aos 8 e 9 anos e competência atlética aos 10 anos. Na subescala comportamental aos 10 e 12 anos o teste $t$ não foi computado, porque a diferença-padrão do erro foi zero (Tabela 4).

Tabela 3

Médias, Correlações e Testes t Pareados nas subescalas da EAPC e nos estudos de Harter (1985) e Van DongenMelman, Koot e Verhulst (1993)

\begin{tabular}{|c|c|c|c|c|c|c|c|}
\hline \multirow{2}{*}{$\frac{\text { Estudos Harter }}{\text { Competência }}$} & \multicolumn{2}{|c|}{ Van Dongen-Melman et al. } & \multicolumn{2}{|c|}{ Presente estudo } & \multicolumn{2}{|c|}{ Presente estudo } & \multirow[b]{2}{*}{$t$} \\
\hline & $M$ & $R$ & $M$ & $R$ & $M$ & $R$ & \\
\hline EAPC total & & - & 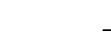 & & 17,38 & $0,83^{*}$ & $-1,46$ \\
\hline Escolar & 2,78 & 0,81 & 2,81 & 0,83 & 2,81 & $0,60 *$ & $-3,29 *$ \\
\hline Aceitação social & 2,80 & 0,75 & 3,08 & 0,80 & 2,75 & $0,54 *$ & $-0,83$ \\
\hline Atlética & 2,84 & 0,80 & 3,07 & 0,78 & 2,74 & $0,60 *$ & $-1,43$ \\
\hline Aparência física & 2,90 & 0,78 & 3,16 & 0,76 & 2,96 & $0,70^{*}$ & 1,22 \\
\hline Conduta comportamental & 2,97 & 0,72 & 2,89 & 0,66 & 2,92 & $0,58 *$ & 1,00 \\
\hline Autoconceito & 2,98 & 0,78 & 3,28 & 0,71 & 3,08 & $0,57^{*}$ & 0,13 \\
\hline
\end{tabular}

Nota. $* p<0,01$.

Tabela 4

Resultados das Correlações Teste-Reteste e Teste t Pareado para cada Domínio da Escala nos Grupos por Idade

\begin{tabular}{lcrrrrr}
\hline \multirow{2}{*}{ Domínio } & Teste & \multicolumn{5}{c}{ Idade em Anos } \\
\cline { 3 - 6 } & & 8 & 9 & 10 & 11 & 12 \\
\hline Escolar & $R$ & $0,53^{*}$ & $0,63^{*}$ & $0,64^{*}$ & $0,60^{*}$ & $0,61^{*}$ \\
& Teste $t$ & $-2,07^{* *}$ & $-4,02^{*}$ & $-0,51$ & 0,00 & 0,25 \\
Aceitação social & $R$ & $0,40^{*}$ & $0,50^{*}$ & $0,57^{*}$ & $0,79^{*}$ & $0,63^{*}$ \\
Atlética & Teste $t$ & $-0,55$ & $-1,92$ & 1,60 & $-0,89$ & $-0,80$ \\
& $R$ & $0,43^{*}$ & $0,66^{*}$ & $0,62^{*}$ & $0,73^{*}$ & $0,48^{*}$ \\
Aparência física & Teste $t$ & $-0,29$ & $-0,65$ & $-2,03^{* *}$ & $-0,49$ & 1,03 \\
& $R$ & $0,50^{*}$ & $0,62^{*}$ & $0,72^{*}$ & $0,77^{*}$ & $0,83^{*}$ \\
Conduta comportamental & Teste $t$ & 1,37 & $-0,19$ & 2,39 & $-1,15$ & $-1,40$ \\
& $R$ & $0,38^{*}$ & $0,54^{*}$ & $0,64^{*}$ & $0,69^{*}$ & $0,62^{*}$ \\
Autoconceito & Teste $t$ & 1,00 & $-0,24$ & $* * *$ & 0,23 & $* * *$ \\
& $R$ & $0,36^{*}$ & $0,59^{*}$ & $0,60^{*}$ & $0,70^{*}$ & $0,70^{*}$ \\
& Teste $t$ & $-0,58$ & $-2,47$ & 0,56 & $-0,92$ & 0,76
\end{tabular}

Nota. $* r_{\text {Pearson }}(p<0,01), * *$ teste $t(p<0,05)$ e *** Não houve variação. 


\section{Análise Fatorial Confirmatória}

Optou-se por verificar a validade da escala para a amostra geral e por sexo. Na amostra geral foram encontrados os seguintes resultados: razão entre o Qui-quadrado e graus de liberdade $(\chi 2 / \mathrm{gl}=4,33) ;$ Non-normed Fit Index $(\mathrm{NFI}=0,958)$; Comparative Fit Index $(\mathrm{CFI}=0,967) \mathrm{e}$ Tucker e Lewis's Index of Fit (LTI = 0,962). Para o sexo masculino obteve-se: razão entre o Qui-quadrado e graus de liberdade $(\chi 2 / \mathrm{gl}=3,00)$; Non-normed Fit Index (NFI $=0,942)$; Comparative Fit Index $(\mathrm{CFI}=0,960)$ e Tucker e Lewis's Index of Fit (LTI = 0,954), e para o sexo feminino, razão entre o Qui-quadrado e graus de liberdade $\left(\chi^{2 / \mathrm{gl}}=3,01\right) ;$ Non-normed Fit Index $(\mathrm{NFI}=0,943) ;$ Comparative Fit Index (CFI = 0,961) e Tucker e Lewis's Index of Fit $(\mathrm{LTI}=0,955)$.

\section{Discussão}

\section{Dupla Tradução Reversa e Independente \\ e Aplicação no Público-Alvo}

A tradução do SPPC por meio da técnica da tradução invertida resultou em uma versão brasileira unificada e final em língua portuguesa, a EAPC. Este processo de tradução reversa (com resolução das diferenças em comissão), pelo rigor dos critérios, permitiu diminuir e até mesmo eliminar as possíveis distorções de uma tradução única (Hernandez-Nieto, 2002; Vallerand, 1989) e fortalecer a validade aparente da escala (Pasquali, 1998). A escala resultante passou pela avaliação do público-alvo com relação à clareza, à propriedade e à adequação para a faixa etária em foco, para certificar-se de que não parecia ridícula para as crianças, já que a opinião de um adulto sobre a clareza de um item para crianças de 8 a 12 anos pode diferir da opinião da própria criança. A tradução e adaptação se mostraram apropriadas para as crianças, de forma a não afetar indiretamente as respostas dos itens da escala (Pasquali, 1998).

\section{A Avaliação dos Juízes}

Os cálculos do Coeficiente de Validade de Conteúdo (CVC) para a clareza e a pertinência foram válidos (Bryman \& Cramer, 1999; Hernandez-Nieto, 2002). Os resultados da correlação intraclasse indicam que o nível de concordância entre os juízes foi alto para a pertinência (Bryman \& Cramer, 1999; Hernandez-Nieto, 2002) das questões da EAPC (todos os resultados superiores a 0,80), mesmo adotando-se índices rigorosos e considerando-se que os juízes avaliadores possuem diferentes formações e experiências profissionais. Os resultados evidenciam a força do instrumento no aspecto pertinência.

Quanto à clareza, os resultados foram próximos ou superiores a 0,70 . Trata-se, neste caso, da opinião dos professores sobre a clareza dos itens na perspectiva de uma criança. Uma posição conservadora, com um julgamento rígido sobre os itens (já que quem deve entender claramente os itens são as crianças, não os juízes), é considerada necessária quanto à clareza na validação de ins- trumentos. Os resultados foram positivos; o único valor um pouco abaixo do desejável $(0,68)$ não se afastou do critério adotado. Estes resultados indicam que a versão em análise da EAPC apresentou índices aceitáveis de validade de conteúdo (Bryman \& Cramer, 1999; Hernandez-Nieto, 2002).

\section{Avaliação da Fidedignidade Teste-Reteste}

As estatísticas evidenciaram que, quanto às questões, em nenhum caso houve adesão aos extremos, o que demonstra a confiabilidade dos valores das médias observadas (entre 2,29 a 3,35), uma vez que estas não sofreram os efeitos negativos da presença de casos, mostrando-se representativas das questões inventariadas. Em nenhum caso ocorreu o fenômeno da aquiescência, quer positiva, quer negativa (Cronbach, 1996; Kline \& Saggino, 1995), o que indica tratar-se de dados confiáveis (Cronbach, 1996). A confiabilidade das médias foi também reportada em vários estudos anteriores com o uso deste instrumento em crianças de outras culturas (Eapen, Naqvi, \& Al-Dhaheri, 2000; Harter, 1985; Miller, 2000; Van Dongen-Melman et al., 1993). Destaca-se que pesquisadores têm reportado médias representativas e confiáveis na validação deste instrumento no estudo com crianças árabes (Eapen et al., 2000), com médias um pouco inferiores $(2,01$ a 2,36$)$ às do presente estudo, e no estudo original de Harter (1985), com crianças americanas (médias entre 2,81 a 3,04).

As correlações teste-reteste permitiram avaliar a fidedignidade do instrumento no que se refere à associação entre variáveis. Os resultados das correlações entre os escores do teste e do reteste na escala total foram positivos, fortes e altamente significativos, atestando a fidedignidade na escala total, semelhante à de estudo anterior com crianças holandesas da mesma, com índices entre 0,66 a 0,86 (Van Dongen-Melman et al., 1993). No presente estudo, as subescalas aparência física e conduta comportamental apresentaram correlações teste-reteste desejáveis, superiores a 0,70 , positivas, fortes e significativas. Duas subescalas (competência escolar e competência atlética) apresentaram correlações teste-reteste um pouco inferiores ao desejável $(r=0,60)$, enquanto duas subescalas (aceitação social e autoconceito) obtiveram correlações teste-reteste inferiores a 0,60 , resultados pouco desejáveis (Berry et al., 1992; Bryman \& Cramer, 1999; Cronbach, 1996; Hernandez-Nieto, 2002; Kline \& Saggino, 1995). Quando estes resultados das correlações são comparados nominalmente com aqueles encontrados por Harter (1985), há uma evidente superioridade na estabilidade temporal nos resultados obtidos pela autora do instrumento, embora os níveis de significância se assemelhem.

A fidedignidade, investigada com relação à concordância numérica por meio do teste $t$ pareado, somente na subescala competência escolar evidenciou diferença significativa $(p<0,05)$ entre teste e reteste no grupo total de crianças (ver Tabela 3). Resultado semelhante 
Valentini, N. C., Villwock, G., Vieira, L. F., Vieira, J. L. L. \& Barbosa, M. L. L. (2010). Validação Brasileira da Escala de Autopercepção de Harter para Crianças.

foi anteriormente reportado por Van Dongen-Melman et al. (1993) com crianças holandesas, as quais apresentaram índices no reteste mais altos que no teste, para a competência escolar. No presente estudo, um exame mais detalhado revela que esta diferença decorre das respostas menos estáveis nas crianças com menor idade (8 ou 9 anos). Este resultado pode ser decorrente de fatores situacionais associados a um julgamento menos maduro das crianças nesta idade. A falta de parâmetros para a avaliação de suas experiências escolares, uma vez que a escola é ainda uma experiência recente para essas crianças (encontram-se nas series iniciais do ensino fundamental), pode ter afetado a estabilidade dos escores entre teste e reteste nas percepções de competência escolar. Van Dongen-Melman et al. (1993) sugerem que a instabilidade nos dados das crianças holandesas de todas as idades quanto à avaliação da competência escolar foi também afetada pelo contexto escolar, mais especificamente pela avaliação de seus desempenhos ocorrida durante as 4 semanas em que o estudo foi realizado. No presente estudo este fator foi controlado e o intervalo teste-reteste, diminuído. Ainda assim a instabilidade se faz presente, embora somente para os mais jovens, o que nos permite inferir que neste domínio as percepções são dependentes do feedback propiciado pela escola e que este, para os mais jovens, ainda pode ser de difícil compreensão. Nas demais subescalas e na escala total, a inexistência de diferenças significativas $(p>0,05)$ evidencia estabilidade temporal, o que é altamente desejável entre os dois momentos avaliativos. Ademais, por se tratar de uma amostra grande, a não-observação de resultados significativos reforça ainda mais a semelhança entre os dois momentos de avaliação (Berry et al., 1992; Bryman \& Cramer, 1999; Vallerand, 1989).

Observa-se que, tanto nas correlações como no teste $t$ pareado, no presente estudo a escala se apresenta com resultados mais satisfatórios nos grupos de idades mais elevadas. Essa tendência foi também reportada no estudo de validação do instrumento nos Emirados Árabes. Entretanto, Eapen et al. (2000) reportam resultados de fidedignidade mais frágeis com crianças árabes entre 8 e 12 anos, sendo que a maior estabilidade no estudo árabe só foi observada para crianças com mais de 12 anos. No presente estudo o instrumento se mostra estável em todas as idades, com a instabilidade restrita à competência escolar antes dos 10 anos. Destaca-se ainda que a estabilidade temporal do instrumento obtida é bastante respeitável quando comparada às de outros instrumentos de autoavaliação (Harter, 1985). Esta estabilidade também assegura que a adaptação dos itens na versão brasileira não aumentou a variação.

\section{Análise Fatorial Confirmatória}

A análise preliminar não evidenciou a existência de "outliers", permitindo o uso da análise factorial confirmatória. O modelo da EAPC foi testado a partir do pa- cote Amos (versão 4.0 para Windows), verificando-se sua adequação (Berry et al., 1992). O modelo proposto (Taylor, Bagby, \& Parker, 2003) considera que cada item deve aferir apenas um fator, diferenciando-se assim do modelo fatorial exploratório, em que cada item apresenta saturações nos diversos fatores com valores próprios superiores a 1. Partindo-se da hipotética associação entre as dimensões do construto avaliado para a amostra (Briggs \& Cheek, 1986) e seguindo-se os procedimentos de validação da autora do instrumento, a adequação do modelo fatorial confirmatório foi testada com o uso do método de estimação Maximuun Likelihood, indicado em grandes amostras, usando-se quatro critérios: razão entre o Qui-quadrado e graus de liberdade, Non-normed Fit Index (NFI), Comparative Fit Index (CFI) e Tucker e Lewis's Index of Fit (TLI). Esses critérios múltiplos foram utilizados porque cada índice apresenta diferentes forças e fraquezas na avaliação da adequação do modelo fatorial confirmatório (Taylor et al., 2003).

Os resultados obtidos na análise fatorial confirmatória para a amostra geral e de sexos para a razão entre Quiquadrado e graus de liberdade foram satisfatórios (todos inferiores a 4,33), uma vez que valores menores que 5 são considerados adequados (Tucker \& Lewis, 1973). Igualmente, as demais medidas de adequabilidade ao modelo, a saber, Non-normed Fit Index (NFI $\leq 0,958)$, Comparative Fit Index (CFI $\leq 0,967)$ e Tucker e Lewis's Index of Fit $(\mathrm{TLI} \leq 0,962)$, estão em acordo com os critérios-padrão, porquanto, segundo a literatura, estes índices devem ser superiores a 0,90 (Tucker \& Lewis, 1973). Os resultados do presente estudo permitem assumir a validade do instrumento para crianças, na amostra pesquisada, tanto para a amostra geral como para os sexos, individualmente. Van Dongen-Melman et al. (1993) reportam índices semelhantes e aceitáveis na adequação do modelo (Goodness-of-fit 0,961; 0,954) com 300 crianças holandesas em idade escolar.

Enfatiza-se que esses índices se alinham aos obtidos em estudos anteriores, que, por meio de testes de fidedignidade e análises factoriais exploratórias e confirmatórias destacam a obtenção de índices aceitáveis de consistência interna e a adequabilidade da escala para mensurar as autopercepções de crianças e adolescentes em culturas bastante diversas. Bandeira et al. (2008) reportaram Chronbach's alphas com valores entre 0,60 a 0,82 nas diversas subescalas e $\alpha=0,88$ na avaliação global no estudo de validação do instrumento de autopercepção para adolescentes realizado também no Sul do Brasil (Santa Catarina e Rio Grande do Sul). Harter (1985), em quatro amostras norte-americanas que envolveram 789 meninas e 732 meninos, reporta índices de Chronbach's alphas entre 0,71 e 0,86. Esses índices são considerados adequados pela literatura, bem como os obtidos com 300 crianças holandesas $(0,65$ a 0,81$)$ no estudo de Van Dongen-Melman et al. (1993) e com os resultados do estudo de Eapen et al. (2000) com 100 crianças dos Emirados Árabes $(0,86$ a 0,92). 


\section{Conclusão}

Este estudo teve por objetivo traduzir para o português o SPPC e verificar as qualidades métricas (fidedignidade teste-reteste, validade de conteúdo e validade fatorial confirmatória) da versão brasileira (EAPC) em uma população de crianças do Paraná e Rio Grande do Sul. O procedimento de tradução invertida mostrou-se eficiente para evitar vieses individuais da compreensão da língua inglesa. Quanto à avaliação das questões por especialistas, a versão brasileira do instrumento evidenciou índices adequados de clareza e elevada pertinência, o que indica tratar-se de instrumento válido, em se tratando do conteúdo, para avaliar as percepções de competência de crianças entre 8 e 12 anos, e que a linguagem da EAPC é de fácil compreensão, no entendimento dos profissionais da área.

Quanto às correlações teste-reteste, na EAPC sugerese cautela na interpretação dos dados referentes à competência escolar em crianças mais jovens (8 e 9 anos), em decorrência da instabilidade observada entre teste e reteste, tendência já apresentada no estudo com crianças holandesas. Entretanto, a correlação teste-reteste da escala total da EAPC não foi afetada por esta instabilidade dos mais jovens nesta subescala específica, assegurando que a mensuração da competência percebida pela criança, avaliada pela EAPC, permaneceu estável ao longo do tempo nas diferentes idades. Ainda mais, observa-se que as subescalas da EAPC são mais consistentes no final da faixa etária em estudo (11 e 12 anos), o que fortalece o próprio modelo teórico proposto por Harter (1999), o qual enfatiza que, com o processo de desenvolvimento cognitivo aliado às experiências vividas, a criança estabelece mais precisamente relações entre suas percepções e a dificuldade das tarefas em que se envolve alterando e estabilizando as suas percepções de competência.

Quanto à análise fatorial confirmatória, verificou-se que a EAPC apresenta índice de validade fatorial confirmatória satisfatório, o que permite assumir a validade do modelo de autopercepção proposto pela versão brasileira na amostra estudada. Há semelhanças entre os dados obtidos por Harter (1985) e os da amostra do presente estudo, quer no tocante ao valor das médias das questões, quer no que tange à validade do modelo. Estes resultados demonstram que este instrumento pode ser aplicado com relativa segurança às crianças brasileiras que possuam características semelhantes às da presente amostra e reforçam as qualidades psicométricas do instrumento em diferentes culturas.

Futuras pesquisas necessitam endereçar questões referentes aos diferentes domínios e sua importância na cultura brasileira. Ademais, faz-se necessário pesquisar se outros itens que não fazem parte da escala seriam relevantes para as percepções de competência escolar, de aceitação social, da competência atlética, da aparência física e da conduta comportamental de crianças brasilei- ras. Destaca-se também a necessidade de investigar como as crianças modificam e estabilizam suas percepções nos diversos domínios do instrumento (escolar, social, atlético, físico, comportamental) e que fatores (contexto sociocultural em que esta inserida, idade, gênero, entre outros) podem influenciar a valorização diferenciada de cada um dos domínios pela criança. Pesquisas são necessárias para o reconhecimento das razões pelas quais as crianças constroem julgamentos próprios sobre competências, da forma como as percepções pessoais intermedeiam as relações com os outros e das interações entre o desenvolvimento social e cognitivo no desenvolvimento singular de cada criança.

\section{Referências}

American Educational Research Association, American Psychological Association, \& National Council on Measurement in Education. (1974). Standards for educational and psychological tests and manuals. Washington, DC: American Psychological Association.

American Educational Research Association, American Psychological Association, \& National Council on Measurement in Education (1999). Standards for educational and psychological testing. Washington, DC: American Educational Research Association.

Altermatt, E. R., \& Pomerantz, E. M. (2003). The development of competence-related and motivational beliefs: An investigation of similarity and influence among friends. Journal of Educational Psychology, 95(1), 111-123.

Bandeira, D. R., Arteche, A. X., \& Reppold, C. T. (2008). Escala de Autopercepção de Harter para Adolescentes: Um estudo de validação. Psicologia: Teoria e pesquisa, 24(3), 341-345

Barber, L., Grubbs, B., \& Cottrell, S. (2005). Self-perception in children with attention deficit/hyperactivity disorder. Journal of Pediatrics Nursing, 20(4), 235-245.

Berry, J. W., Poortinga, Y. H., Segall, M. H., \& Dasen P. R. (1992). Cross-cultural Psychology: Research and applications. Cambridge, MA: Cambridge University Press.

Briggs, S. R., \& Cheek, J. M. (1986). The role of factor analysis in the development and evaluation of personality scales. Journal of Personality, 54(4), 106-148.

Bryman, A., \& Cramer, D. (1999). Quantitative data analysis with SPSS release perceived symptoms - Criterion validity of the Toronto Alexithymia LOS8 for Windows: A guide for social scientists. New York: Routledge.

Chen, X., Zappulla, C., Lo Coco, A., Schneider, B., Kaspar, V., Oliveira, A. M., et al. (2004) Self-perceptions of competence in Brazilian, Canadian, Chinese and Italian children: Relations with social and school adjustment. International Journal of Behavior Development, 28(2), 129-138.

Chernoff, R. G., Ireys, H. T., DeVet, K. A., \& Kim, Y. J. (2002). A randomized, controlled trial of a community-based Support Program for Families of Children with Chronic Illness: Pediatric Outcomes. Archives of Pediatrics and Adolescence Medicine, 156(6), 533-539.

Cronbach, L. J. (1996). Fundamentos da testagem psicológica. Porto Alegre, RS: Artes Médicas.

Dassa, C. (1999) Analyze multidimensionnelle exploratoire et confirmative. Montreal, Canada: Univesité de Montreal. 
Donnellan, M. B., Trzesniewski, K. H., Robins, R. W., Moffitt, T. E., \& Caspi, A. (2005). Low self-esteem is related to aggression, antisocial behavior, and delinquency. Psychology Science, 16(4), 328-335.

Eapen, V., Naqvi, A., \& Al-Dhaheri, A. S. (2000). Cross-cultural validation of Harter's Self-Perception Profile for Children in the United Arab Emirates. Annals of Saudi Medicine, 20(1), 8-11.

Fiorese, L. (1993). A relação entre a percepção de competência de atletas adolescentes e seus motivos para a prática esportiva. Revista da Educação Física/UEM, 4(1), 40-50.

Franklin, J., Denyer, G., Steinbeck, K. S., Caterson, I. D., \& Hill, A. J. (2006). Obesity and risk of low self-esteem: A statewide survey of Australian Children. Pediatrics, 118(6), 2481-2487.

Harter, S. (1985). Manual for the self-perception profile for children. Denver, CO: University of Denver.

Harter, S. (1999). The construction of the self: A developmental perspective. New York: Guilford Press.

Hassandra, M., Goudas, M., \& Chroni, S. (2003). Examining factors associated with intrinsic motivation in physical education: A qualitative approach. Psychology Sport Exercise, 4(1), 211-223.

Heath, N. L., \& Glen, T. (2005). Positive illusory bias and the self-protective hypothesis in children with learning disabilities. Journal of Clinical Child Adolescence Psychology, 34(2), 272-281.

Hernandez-Nieto, R. (2002). Contributions to statistical analysis. Mérida, España: Los Andes University Press.

Kline, P., \& Saggino, A. (1995). Item factor analysis of the Italian version of the Myers-Briggs Type Indicator?Source. Personality Individual Differences, 19(2), 243-249.

Miller, H. M. (2000). Cross-cultural validity of a model of self-worth: Application to finnish children. Social Behavior Personality, 28(2), 105-118.

Pasquali, L. (1998). Princípios de elaboração de escalas psicológicas [Special issue]. Revista de Psiquiatria Clínica, 25(5), 206-213.

Pasquali, L. (1999). Instrumentos psicológicos: Manual prático de elaboração. Brasília, DF: Laboratório de Pesquisa em Avaliação e Medida.

Pasquali, L. (2003). Psicometria: Teoria dos testes na Psicologia e na Educação. Petrópolis, RJ: Vozes.

Taylor, G. J, Bagby, R. M., \& Parker, J. D. A. (2003). The 20Item Toronto Alexithymia Scale IV. Realiability and factorial validity in different languages and cultures. Journal of Psychology Research, 55(2), 277-283.

Tucker, S., \& Lewis, C. (1973). A reliability coefficient for maximum likelihood factor analysis. Psychometrika, 38(1), 1-10.

Urbina, S. (2004). Essentials of psychological testing. Hoboken, NJ: John Wiley \& Sons.

Valentini, N. C., \& Rudisill, M. E. (2004). An inclusive Mastery Climate Intervention on the motor skill development of children. Adapted Physical Activity Quarterly, 21(4), 330347.

Vallerand, J. R. (1989).Vers une mèthodologie de validation trans-culturelle de questionnaires psychologiques: implications pour la recherche en langue fraçaise. Canadian Psychology, 30(4), 662-680.

Van Dongen-Melman, J. E. W. M., Koot, H. M., \& Verhulst, F. C. (1993). Cross-cultural validation of Harter's Self-Perception Profile for Children in a Dutch Sample. Educational Psychology Mesurament, 53(3), 739-753.
Van Pareren, Y. K., Duivenvoorden, H. J., Slijper, F. S. M., Koot, H. M., \& Hokken-Koelega, A. C. S. (2004). Intelligence and psychosocial functioning during Long-Term Growth Hormone Therapy in Children Born Small for Gestational Age. Journal of Clinical Endocrinology Metabolism, 89(11), 5295-5302.

Vieira, L. F., Vieira, J. L. L., \& Krebs, R. J. (1997). Análise da percepção de competência no contexto escolar. Revista Kinesis, 4(15), 24-30.

$\mathrm{Wu}$, X. (2003). Intrinsic motivation and young language learners: The impact of the classroom environment. System Development, 31(1), 501-517. 